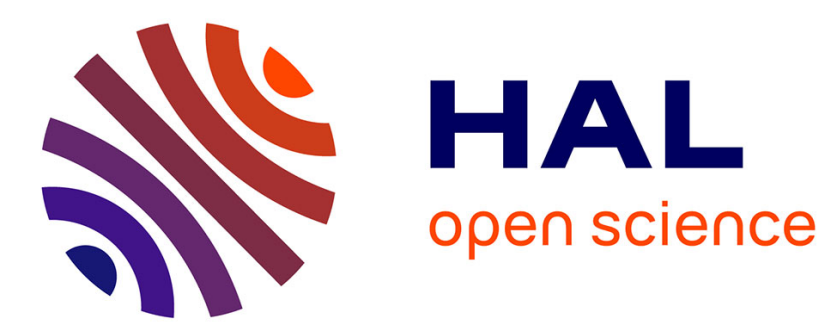

\title{
Blocking Evaluation and Wavelength Dimensioning of Dynamic WDM Networks without Wavelength Conversion
}

\author{
Nicolas Jara, Reinaldo Vallejos, Gerardo Rubino
}

\section{- To cite this version:}

Nicolas Jara, Reinaldo Vallejos, Gerardo Rubino. Blocking Evaluation and Wavelength Dimensioning of Dynamic WDM Networks without Wavelength Conversion. Journal of Optical Communications and Networking, 2017, 9 (8), pp.625-634. 10.1364/JOCN.9.000625 . hal-01663506

\author{
HAL Id: hal-01663506 \\ https://hal.inria.fr/hal-01663506
}

Submitted on 14 Dec 2017

HAL is a multi-disciplinary open access archive for the deposit and dissemination of scientific research documents, whether they are published or not. The documents may come from teaching and research institutions in France or abroad, or from public or private research centers.
L'archive ouverte pluridisciplinaire HAL, est destinée au dépôt et à la diffusion de documents scientifiques de niveau recherche, publiés ou non, émanant des établissements d'enseignement et de recherche français ou étrangers, des laboratoires publics ou privés. 


\title{
Blocking Evaluation and Wavelength Dimensioning of Dynamic WDM Networks without Wavelength Conversion
}

\author{
Nicolás Jara, Reinaldo Vallejos and Gerardo Rubino
}

\begin{abstract}
In this paper, a new fast and accurate methodology to evaluate the blocking probability (burst loss probability) in dynamic WDM networks without wavelength conversion is presented. The proposed model considers different traffic loads at each network connection (heterogeneous traffic). To take into account the wavelength continuity constraint, the method sees the network as a sequence of networks where all the links have capacity 1. Every network in the sequence is evaluated separately using an heuristic-based analytical technique. Then, a procedure combines the results of these evaluations in a way that captures the dependencies that occur in real systems due to the competition for bandwidth between the different connections. The method efficiently achieves results very close to those obtained by simulation, but orders of magnitude faster, allowing the evaluation of the blocking probability of all users (connections) for mesh network topologies. We illustrate the use of this procedure in the dimensioning of a WDM network, that is, in calculating the number of wavelengths on every network link, by considering the traffic load of every user, the routing algorithm and the maximum connection blocking probability acceptable by every user (quality of service).
\end{abstract}

Index Terms-Blocking Probability, Dynamic WDM Optical Networks, No Wavelength Conversion, Wavelength Dimensioning, Heterogeneous traffic.

\section{INTRODUCTION}

$\mathbf{T}$ HE rapid increase in demand for bandwidth on existing networks has caused a growth in the use of technologies based on WDM optical infrastructures [1], [2]. Currently, this type of network is operated statically [1], i.e., the resources used by a connection (user) is permanently assigned from source to destination. This type of operation is inefficient in the usage of network assets, specially for low traffic loads, which is the most common case.

One way to help overcome these inefficiencies is to migrate them to networks working dynamically. This operation mode consists in allocating the resources required only when the user has data to transmit. A possible lack of resources to successfully transmit can then happen, because dynamic networks are designed to save costs using the less possible

N. Jara is with the Electronic's Department in Universidad Técnica Federico Santa Maria (UTFSM), Valparaíso, Chile and INRIA Rennes Bretagne Atlantique, Rennes, France e-mail: nicolas.jara@usm.cl

R. Vallejos is with the Electronic's Department in Universidad Técnica Federico Santa Maria (UTFSM), Valparaíso, Chile e-mail: reinaldo.vallejos@usm.cl

G. Rubino is with INRIA Rennes - Bretagne Atlantique, Rennes, France e-mail: gerardo.rubino@inria.fr

Manuscript received; revised. amount of resources, and simultaneously to be effective (low burst losses). To achieve a tradeoff between these two contradictory aspects, the network must be designed such that the connection blocking probability is less than or equal to a design parameter $\beta$. The evaluation of the real blocking probability achieved allows to determine whether or not each network user (each connection) is being treated with the required quality of service. As a result, the blocking probability is one of the main parameters that has been used to evaluate the performance of dynamic WDM optical networks [3].

In general, the blocking probability is evaluated through simulation [4]-[6]. The reason is that the mathematical analysis is most of the time out of reach, because of the complexity of the analysis, the combinatorial explosion problem, etc. Nevertheless, simulations are in general very slow compared with the solution obtained following a mathematical approach [7]. The evaluation speed is relevant, because when solving problems of higher order (e.g. concerning routing or fault tolerant mechanisms), it is in general necessary to calculate the blocking probability a large number of times. Thus, a fast and accurate mathematical computational method is extremely useful. However, to obtain a mathematical procedure with such characteristics is a difficult task, due to important aspects to take into account while modeling, such as: traffic load, wavelengths capacity, wavelength continuity constraint (because the network operates without wavelength conversion), network topology, etc. Therefore, several hypotheses are typically introduced to simplify the model in order to facilitate its analysis.

One of these hypotheses is the homogeneous load assumption. Many works assume that the traffic load offered by each connection to the network is statistically the same [8][15]. This hypothesis strongly simplifies the modeling, but it does not adequately represent the operation of optical networks (or computer networks in general), because the offered traffic is usually very heterogeneous. This is relevant since replacing each of the sources by the average of all of them can significantly modify the performance metrics of the system [16]. This underlines the interest in including the traffic load heterogeneity on the network mathematical models used. In [17] a model based on the Erlang-B formula is proposed with the purpose of evaluating the link blocking probability. This model allows different traffic loads on each 
network link, but since it is based on the Erlang-B formula, the individual loads don't appear in the solutions (only their sums do), and thus, it suffers from the same limitations as when the homogeneous assumption is used.

Another commonly used hypothesis is the Poisson traffic assumption, shared by the majority of papers published so far [8]-[15], [17]-[19], which greatly simplifies the mathematical evaluation. However, a Poisson process is not representative of the real traffic in optical networks, for several reasons. For instance, the rate of the offered traffic in a given link varies significantly over time, because it is sensitive to the number of connections that are not currently transmitting. Another way of using de Poisson modelling was proposed in [8], [9], [13] where the network is split into several layers (one for each wavelength) where the blocked traffic in one layer is overflowed to the next. This overflowed traffic its not Poisson (it is bursty), therefore the author uses the Fredericks and Hayward's approximation [20], [21] to transform the bursty overflowed traffic (non-Poisson) into a Poisson flow. The solving procedure then applies the Erlang-B formula separately at each layer to solve their blocking probability. This formula can be used on queuing systems where the arrival rate does not change on time, which happens when there is a huge number of connections. However, in an optical network the total number of users that can share a network link is low, and the arrival rate (on any instant and any network link) depends on the number of active connections passing through the link. Then, the arrival rate changes significantly over time, making this model inadequate.

In this paper we propose a new approach to evaluate the blocking probability (of burst losses) in dynamic WDM optical networks without considering wavelength conversion and with heterogeneous traffic. The method is called Layered Iterative Blocking Probability Evaluation, LIBPE in the text. It takes into account the bursty nature of the offered traffic, by modeling the sources with ON-OFF processes. Our technique obtains very accurate results in comparison to those achieved by simulation, with computational speed orders of magnitude faster.

We illustrate the use of our technique for calculating the number of wavelengths on every network link, that is, for dimensioning the WDM network. The final network dimensioning results show that the proposed method obtains the same results as the ones obtained by simulation (which in general are based on the sequential execution of simulation experiments), but much faster (e.g. between $10^{3}$ and $10^{4}$ times faster).

The remainder of this paper is as follows: In Section II we use a layer-based strategy to evaluate the blocking probability. Section III presents some numerical examples. Then, Section IV] presents the dimensioning method and the obtained results. Finally, conclusions are given in Section $\mathrm{V}$.

\section{BLOCKING EVALUATION STRATEGY}

The network is represented by a directed graph $\mathcal{G}=(\mathcal{N}, \mathcal{L})$, where $\mathcal{N}$ is the set of network nodes and $\mathcal{L}$ is the set of unidirectional links (the graph's arcs), with respective cardinalities $N$ and $L$. The set of connections (or users) $\mathcal{C} \subseteq \mathcal{N}^{2}$, with cardinality $C$, is composed by all the source-destination pairs with communication between them, together with the route followed by the data.

To represent the traffic between a given source-destination pair an ON-OFF model is used. In works such as [22], it has been demonstrated that the blocking probability on dynamic networks is mainly affected by the mean times $t_{O N} \mathrm{y} t_{O F F}$, and is practically insensitive to the specific distribution of such times. In fact, in [23], the sensitivity of the blocking probability in dynamic networks on the blocking probability was studied in such networks. That work concluded that, for practical purposes, we can consider this probability as insensitive to the specific distribution of $t_{O N} \mathrm{y} t_{O F F}$. Consequently, to represent the times of formation and transmission of bursts, our work uses only the mean values of those times.

Consider connection $c$. During any of its ON periods, whose average length is $t_{O N}$, the source transmits at a constant transmission rate. During an OFF period, with average length $t_{O F F_{c}}$, the source refrains from transmitting data. We use the notation $\tau_{c}=t_{O N_{c}}+t_{O F F_{c}}$, and call it the average length of a cycle for connection $c$. For a given user, we assume that the lengths of ON (respectively OFF) periods are i.i.d. random variables, and that both sequences are independent of each other.

When traffic sources are $\mathrm{ON}$, they all transmit at the same rate, determined by the used technology, that to simplify the presentation will be our rate unity. Consequently, the traffic load for connection $c$, denoted by $\varrho_{c}$, given by the following expression,

$$
\varrho_{c}=\frac{t_{O N_{c}}}{t_{O N_{c}}+t_{O F F c}},
$$

is also the mean traffic offered by connection $c$.

Let $\mathcal{R}=\left\{r_{c} \mid c \in \mathcal{C}\right\}$ be the set of routes that enable communication among the different users, where $r_{c}$ is the route associated with connection $c \in \mathcal{C}$. To simplify the explanation, we assume for the moment that every link $\ell \in \mathcal{L}$ has a same number $W$ of wavelengths associated with, but keep in mind that our method allows different number of wavelengths on each network link (see at the end of Section II, paragraph $a$ ).

Let the $W$ available wavelengths be numbered $1,2, \ldots, W$. The network basically operates as follows. Upon the arrival of a connection request to destination $d$, say by user $c$, the source $s$ will attempt to transmit on the first wavelength $w=1$ on the predetermined fixed path from node $s$ to node $d$, by assuming a "first fit" wavelength allocation method. The request is accepted if wavelength 1 is available on all the links belonging to the predetermined fixed path (wavelength continuity constraint), that is, on route $r_{c}$. Otherwise, the same request is offered to the next wavelength $(w=2)$. The process continues in the same way, until there is some wavelength available on all the links of the path, or until the $W$ wavelengths have been 
considered and none was available along the path. In the first case, the node $s$ transmits its information to $d$ through the available wavelength, and in the last case, the request is blocked (lost).

Given the complexity of the exact evaluation of the blocking probability considering all the aspects described before, we developed a strategy to obtain an accurate while light cost approximate computational scheme. Note that one of the most important aspects to consider is the wavelength continuity problem, because there is no wavelength conversion capability. This means that when a connection transmits, it must use the same wavelength on each link that belongs to its route. We explain below the different steps of the LIBPE procedure.

\section{A. Auxiliary sequence of networks}

Observe that, from the vocabulary point of view, we can consider that the network is actually composed of $W$ networks operating "in parallel", with the same topology as the original one, which we denoted by $\mathcal{G}$, but where each link has a single wavelength associated with (that is, a capacity equal to 1 ). We will see this set of networks as a sequence $\left\langle\mathcal{G}_{1}, \mathcal{G}_{2}, \ldots, \mathcal{G}_{W}\right\rangle$ and say that these auxiliary networks are different "layers" of $\mathcal{G}$. Moreover, the single wavelength associated with each link in $\mathcal{G}_{w}$ is precisely the one having number $w$. Then, an arriving connection will look for room in layer 1 first, if this fails, in $\mathcal{G}_{2}$, and so on, until it finds available capacity in one of the $W$ layers, or until all of them block it.

The technique proposed in this work will then follow a decomposition approach: each layer will be analyzed in isolation, but its parameters will depend on what happens on the other layers. The heuristic mentioned in the abstract appears in the way these two elements (solving for a layer and using the dependencies between them) are treated.

To take into account the interaction between the $W$ auxiliary networks we will establish a dependency between the mean lengths of the OFF periods associated with the sources. That is, the traffic offered to the different layers in the analysis process will (naturally) be different for each one, and its calculation will take into account the different ways where what happens with a wavelength impacts the traffic that will arrive to another one.

In the following, we describe the procedure in detail, which constitute the main contribution of the paper.

\section{B. Network analytical model when $W=1$}

Since the network is divided into a sequence of $W$ networks/layers where each link has now capacity 1 , we consider first the case of $W=1$. With this, we have a method to solve any of the $\mathcal{G}_{w}$ networks generated on the network division with link capacity equals to 1. Fix a link in the network, say link $\ell$. Some connections (at least one) use this link in their routes, some don't. Denote by $T_{\ell}$ the number of connections using $\ell$, and assume that, once $\ell$ fixed, we renumber the connections so that those using link $\ell$

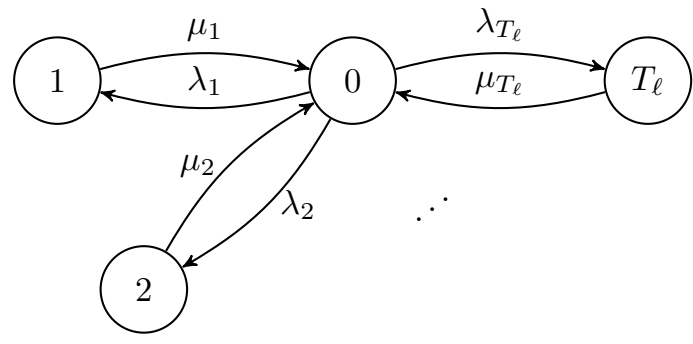

Figure 1. Markov chain modeling the occupation of a given link in a network where all links have only one wavelength. There are $T_{\ell}$ connections using the link. State $c$ means that connection $c$ is using the link, $c=1,2, \ldots, T_{\ell}$. State 0 means that the single wavelength of the link is available. Arrival rate of a burst of connection $c: \lambda_{c}=1 / t_{O F F c}$. Service rate (by the link) of a burst of connection $c$ : $\mu_{c}=1 / t_{O N}$.

are $1,2, \ldots, T_{\ell}$. Observe that link $\ell$ can be either free, or busy transmitting a burst from connection $c, c=1,2, \ldots, T_{\ell}$.

Assume the system is in equilibrium, and denote by $B L_{c, \ell}$ the blocking probability of connection $c$ at link $\ell$, that is, the probability that a burst of connection $c$ arriving at link $\ell$ finds it busy. To evaluate it, assume Markovian conditions, that is, Exponentially distributed burst generation times and Exponentially distributed burst transmission times, with respective rates $\lambda_{c}$ and $\mu_{c}$, and add the usual independence conditions. The service rate $\mu_{c}$ is simply $\mu_{c}=1 / t_{O N_{c}}$. For the arrival rate, observe first that when the link ends transmitting a burst, all the $T_{\ell}$ connections (including the one that just transmitted) are in the OFF part of their cycles (this is because we are dealing with a loss system). So, when entering state 0 , we have $T_{\ell}$ exponential clocks competing, the $c$-th one with an exponentially distributed time length having parameters $1 / t_{O F F_{c}}$. So, $\lambda_{c}=1 / t_{O F F_{c}}$. The continuous time stochastic process $Z=\{Z(t), t \geq 0\}$ on the state space $\left\{0,1,2, \ldots, T_{\ell}\right\}$, representing the state of the link at time $t$ is then Markov (see Figure 11). A straightforward analysis of this Markov chain gives its steady state distribution $\left(\pi_{0}, \pi_{1}, \ldots, \pi_{T_{\ell}}\right)$. The equilibrium equation of state $c \in\left\{1,2, \ldots, T_{\ell}\right\}$ is

$$
\pi_{c} \mu_{c}=\pi_{0} \lambda_{c}
$$

This immediately leads to

$$
\pi_{c}=\frac{\phi_{c}}{1+\phi}, \quad c=1,2, \ldots, T_{\ell}, \quad \pi_{0}=\frac{1}{1+\phi},
$$

where $\phi_{c}$ is the ratio $\phi_{c}=\lambda_{c} / \mu_{c}=t_{O N_{c}} / t_{O F F_{c}}$ and $\phi$ is the sum $\phi=\phi_{1}+\cdots+\phi_{T_{\ell}}$. Observe that, in terms of loads, we have $\phi_{c}=\varrho_{c} /\left(1-\varrho_{c}\right)$, since $\varrho_{c}=\lambda_{c} /\left(\lambda_{c}+\mu_{c}\right)$.

It is immediate to see that the equilibrium distribution (3) doesn't depend on the distribution of ON periods, that is, it doesn't change if the ON periods have any other distribution with finite expectation if this expectation is equal to $1 / \mu_{c}$ for user $c$. The reason is that any state $i \neq 0$ has only one successor (state 0 ), so, the model's stationary distribution doesn't change if we set the distribution of any of these holding times to another distribution with the 
same mean (see, for instance, [24, Prop. 4.8.1] on semiMarkov processes). Here, for simplicity in the presentation, we assume Exponential ON times.

The blocking probability $B L_{c, \ell}$ is the ratio between the probability of a connection $c$ request being blocked for lack of resources and the probability of the union of all possible scenarios when connection $c$ wants to transmit. It can also be derived marking connection $c$ arrivals and analyzing the chain embedded at the marked transition epochs. The result is

$$
B L_{c, \ell}=\frac{1-\pi_{0}-\pi_{c}}{1-\pi_{c}}=\frac{\phi-\phi_{c}}{1+\phi-\phi_{c}} .
$$

Since we are considering this evaluation on any of the $\mathcal{G}_{w}$ networks, we can conclude that the link blocking probability on the w-th network $B L_{c, \ell}^{w}$ is equal to the one obtained on equation 4 considering the $\mathcal{G}_{w}$ network values $t_{O N}$ and $t_{O F F}$.

The blocking probability of connection $c$, with $c \in \mathcal{C}$, on network $\mathcal{G}_{w}$, that is, the probability that a burst of connection $c$ arriving at $\mathcal{G}_{w}$ finds at least one link busy in its route, can be then approximated by means of the typical link independence assumption, that is, by assuming that the states of the links in the network (or just in the route) are independent of each other. This gives

$$
B C_{c}^{w}=1-\prod_{\ell \in r_{c}}\left(1-B L_{c, \ell}^{w}\right) .
$$

This independence assumption is not realistic in this highly competitive context where many connections can be often trying to access simultaneously the same resources (this is called "Streamline Effect" in [25]). Moreover, remember that in the first $\mathcal{G}_{w}$ networks, with $w$ close to 1 , we are considering the border case where resources are really scarce (there is only one wavelength per link, and several users trying to use it). To improve the quality of the approximation, we use the fixed point method proposed by Kelly [26]: once $B C_{c}$, for all connections $c$, is computed, we modify the arrival rate $\lambda_{c}$ by replacing it with the value $\lambda_{c}^{\prime}=\lambda_{c}\left(1-B C_{c}\right)$. Then, we recompute a new blocking probability $B C_{c}^{\prime}$ for all $c$, and we repeat the process until this convergence criteria is satisfied.

\section{Networks interaction}

Now that every layer performance can be evaluated using the scheme described in II-B, we need to take into account the interaction between each of the $W$ networks $\mathcal{G}_{1}, \ldots, \mathcal{G}_{W}$. This interaction will be captured through new values characterizing the ON-OFF arrival processes of the different connections on each network $\mathcal{G}_{w}$. For this purpose, let us denote by $t_{O N_{c, w}}$ and $t_{O F F_{c, w}}$ the average values of $\mathrm{ON}$ and OFF periods for connection $c$ in network $\mathcal{G}_{w}$. Regarding $t_{O N_{c, w}}$, since it is the time used by source $c$ to transmit, it will be kept equal to the initial data $t_{O N_{c}}$. In other words, the dependencies between layers will be captured only by the $t_{O F F_{c, w}}$ values and for all source $c$ and wavelength $w$, $t_{O N_{c, w}}=t_{O N_{c}}$.
As a way of example, figure 2 shows the time system diagram seeing by the user 1 when there are 4 users and a link network capacity of 3 wavelengths (Each network named $\mathcal{G}_{1}, \mathcal{G}_{2}$ and $\mathcal{G}_{3}$ ). The upper part (first 3 horizontal lines of the figure), shows how the connections use the wavelengths resources, and how the user 1 sees them work on any wavelengths separately in different scenarios, represented by its $t_{O N}$ and $t_{O F F}$. The lower part (under the dotted horizontal line) shows how the user 1 destination node "sees" the overall transmission time. Every arrival its marked with a vertical arrow. If the connection request is blocked on a wavelength, then an X appears on the bottom of the arrow, and if the connection is accepted a block corresponding a transmission time $\left(t_{O N}\right)$ appears. As mention before wavelength allocation is First Fit as can be seen on the figure diagram.

The heart of our procedure concentrates then on those dependencies between layers, which are of three types, informally described below and represented on the Time Equivalence Diagram shown on figure 2. Then, the precise way in which these interactions will be taken into account in the equations will be explicitly presented.

- Sequential dependency: when a request from connection $c$ is accepted at layer $\mathcal{G}_{w}$, the next layers will not receive it. In other words, a wavelength in the sequence receives a request from connection $c$ only if it is blocked in every previous wavelengths in the sequence. Therefore, $t_{O F F_{c, w^{\prime}}}$ will grow by the quantity $\tau_{c}=t_{O F F_{c}}+t_{O N_{c}}$ in every $w^{\prime}>1$, for each request transmitted on the wavelengths previous to $w^{\prime}$. This can be seen on figure 2, where the second wavelength will receive a request only when the same connection request is blocked on the first wavelength..

- Backward dependency: After connection $c$ is blocked on network $\mathcal{G}_{1}$ and accepted in any of the next wavelengths, the next transmission request (in $\mathcal{G}_{1}$ ) of connection $c$ will be after one transmission period -because the first request was accepted- and one idle period (mean length $t_{O F F_{c}}$ ) to recollect new data to transmit. Therefore, all blocked connections in network $\mathcal{G}_{1}$, but accepted on any of the next networks $\mathcal{G}_{w}, w>1$, make $t_{O F F_{c, 1}}$ grow by $\tau_{c}$. Notice that this does not affects only on the first layer $\mathcal{G}_{1}$, but every layer on the network. But, it is enough to consider this dependency only on $\mathcal{G}_{1}$, because the sequential dependency will spread this effect. This can be seen on figure 2, where the user 1 next request time on the first wavelength increases every time user 1 is accepted on any other wavelength but the first.

- General blocking dependency: This considers the scenario when a connection $c$ request is blocked on every wavelength (every network $\mathcal{G}_{w}$, for all $w$ ). In this case, connection $c$ start again to recollect new data to transmit (OFF period). Therefore, all blocked connections in the final network $\mathcal{G}_{W}$ make the $t_{O F F_{c, 1}}$ value increase by $t_{O F F_{c}}$. This can be seen in fig. 2 when the user 1 is 


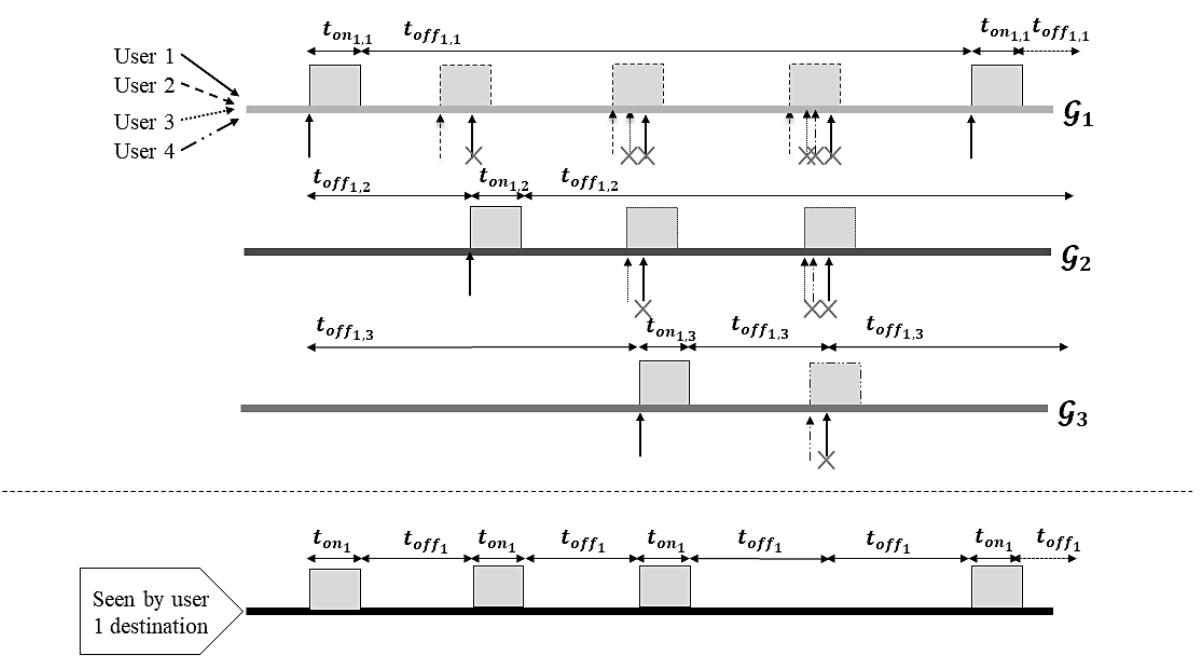

Figure 2. Time Equivalence Diagram. This figure shows every possible scenario where the user 1 can be accepted or blocked when making a connection request. The network has 4 users and a link's capacity of 3 wavelengths. The upper half (above the dotted line) corresponds to each one of the 3 wavelengths showing the real $t_{O F F 1}$ and $t_{O N 1}$ seen on each wavelength by the user 1 and the lower part (bellow the dotted line) shows how the user 1 times are taking place.

\section{blocked on every wavelength.}

Let us go now through the details concerning the evaluation of the $t_{O F F_{c, w}}$ on every wavelength, considering the dependencies just introduced. For this purpose, we will denote by $B C_{c}^{w}$ the blocking probability of connection $c$ at layer $\mathcal{G}_{w}$.

- First Wavelength $(w=1)$. On the first wavelength, that is, on the first layer $\mathcal{G}_{1}$, we take into account the last 2 dependencies. First, when connection $c$ is blocked on $\mathcal{G}_{1}$ and accepted in any of the next layers, it will have to wait an additionally delay $\tau_{c}$ until trying to transmit again on $\mathcal{G}_{1}$ (Backward dependency). Second, if the connection is blocked in every following wavelength, that is, in every layer, then the connection starts again an OFF period (general blocking dependency). These facts translates into the following relationship, by considering every possible scenario in a probabilistic way:

$$
\begin{aligned}
t_{O F F_{c, 1}}=t_{O F F_{c}} & \\
& +0 \cdot\left(1-B C_{c}^{1}\right) \\
& +\tau_{c} B C_{c}^{1}\left(1-B C_{c}^{2}\right) \\
+ & \tau_{c} B C_{c}^{1} B C_{c}^{2}\left(1-B C_{c}^{3}\right) \\
+\cdots & \\
+ & \left(\prod_{c}^{W-1} B C_{c}^{k}\right)\left(1-B C_{c}^{W}\right) \\
& +t_{O F F_{c}}\left(\prod_{k=1}^{W} B C_{c}^{k}\right) .
\end{aligned}
$$

The first term in 6 considers the source $t_{O F F_{c}}$ of the connection $c$. The second term represents the probability of being accepted on the first wavelength, in which case the value of $t_{O F F_{c, 1}}$ remains unchanged. The next terms correspond to the cases of connection $c$ accepted at the next layers $(w>1)$, in which case applies the Backward dependency. The very last term handles the case where the connection is blocked on every possible wavelength, then applies the general blocking dependency. Now, (6) can be simplified into

$$
\begin{aligned}
t_{O F F_{c, 1}}= & t_{O F F_{c}}\left(1+\prod_{k=1}^{W} B C_{c}^{k}\right) \\
& +\tau_{c} \sum_{1 \leq i \leq W-1}\left(1-B C_{c}^{i+1}\right) \prod_{k=1}^{i} B C_{c}^{k} .
\end{aligned}
$$

The last term in previous equation has a telescopic sum inside. This allows a further simplification:

$$
t_{O F F_{c, 1}}=t_{O F F_{c}}+\tau_{c} B C_{c}^{1}-t_{O N} \prod_{k=1}^{W} B C_{c}^{k}
$$

- Next wavelengths now. Observe first that layer $\mathcal{G}_{w}$, for $w>1$, will not have a connection request from user $c$ until this user is blocked on all previous layers. So, the connection $c$ mean OFF period length increase a mean cycle length $\tau_{c}$ for every time the user $c$ was accepted on any of the previous layers.

Consider $B C_{c}^{m}$, with $m<w$, the blocking probability of connection $c$ on $\mathcal{G}_{m}$. Notice that the periods between consecutive successful transmissions on $\mathcal{G}_{m}$, are statistically equivalent. Then, the probability that a sequence of transmission request are successful, is given by a sequence of random variable i.i.d. Bernoulli, with success parameter equals to $1-B C_{c}^{m}$. This implies that the mean number of connection $c$ request in network $\mathcal{G}_{m}$ until the first one is blocked is $\frac{1}{B C_{c}^{w}}$. Therefore, the mean number of user $c$ successful transmissions on network $\mathcal{G}_{m}$ is equal to $\frac{1}{B C_{c}^{w}}-1$. This happens on each 
previous network previous to $\mathcal{G}_{w}$, therefore it must be considered all of them.

Then, the mean length of the OFF period corresponding to connection $c$ for wavelength $w>1$ is

$$
t_{O F F_{c, w}}=t_{O F F_{c, w-1}}+\tau_{c} \sum_{m=1}^{w-1}\left(\frac{1}{B C_{c}^{m}}-1\right) .
$$

This equation shows how the mean time off $t_{O F F_{c, w}}$ seen on the $\mathcal{G}_{w}$ network captures the sequential overflow traffic of every connection $c$.

\section{Network blocking evaluation}

The total network blocking probability of a dynamic WDM network (that is, the blocking probability of an arriving burst, without considering to which connection it belongs), $B_{n e t}$, will be measured by the total blocked burst rate divided by the total burst rate arriving at the network, i.e.,

$$
B_{n e t}=\frac{\sum_{c \in \mathcal{C}} \varrho_{c} B C_{c}}{\sum_{c \in \mathcal{C}} \varrho_{c}},
$$

where $B C_{c}$ is the overall blocking probability of connection $c$, calculated by

$$
B C_{c}=\prod_{\text {all } w} B C_{c}^{w}
$$

It is important to note that the evaluation of the $B C_{c}^{w}$, for all $\mathcal{G}_{w}$, requires the values of $t_{O F F_{c, w}}$ for all $w$. On the other hand, according to the relationships (8) and (9), the value of every $t_{O F F_{c, w}}$ requires the values of $B C_{c}^{w}$, for all $\mathcal{G}_{w}$. This problem can then be solved with an iterative fixed point method, where $B C_{c}^{w}$, for all $\mathcal{G}_{w}$, is initially set to 0 .

The corresponding pseudo-code of the entire strategy is summarized in Algorithm 1. In this pseudo-code five functions are used.

- Initialize $(c)$ : This function assigns the respective values of $t_{O N_{c}}$ and $t_{O F F_{c}}$ to connection $c$, for all $c \in \mathcal{C}$.

- $\operatorname{LinkBP}(\ell)$ : For a given network $\mathcal{G}_{w}$, this function evaluates the link blocking probability of every connection $c$ passing through link $\ell$, using Equations (3) and (4).

- ConnectionBP $(c)$ : For a given network $\mathcal{G}_{w}$, this function evaluates the connection $c$ end-to-end blocking probability using Equation (5).

- NetworkBP(): This function evaluates the global network blocking probability using Equation (10).

- Update $\left(t_{O F F_{c}}, w, j\right)$ : This function updates the mean time OFF "seen" by network $\mathcal{G}_{w}$ on iteration $j$, i.e. $t_{O F F_{c, w}}$, as explained before (Equations (8) and (9)).

a) Case of different numbers of wavelengths in different links: assume now that link $\ell$ of $\mathcal{G}$ has capacity $\mathcal{W}_{\ell}$, that is, can work with wavelengths $1,2, \ldots, \mathcal{W}_{\ell}$, where the $\mathcal{W}_{\ell} \mathrm{s}$ are not necessarily the same on each link. To handle this general case, we divide the network into $\mathcal{W}_{\max }$ layers, where $\mathcal{W}_{\max }=\max _{\ell \in \mathcal{L}}\left\{\mathcal{W}_{\ell}\right\}$. As before, all links in each layer has a single (and the same) wavelength associated with. If link $\ell \in \mathcal{L}$ has capacity $\mathcal{W}_{\ell}<\mathcal{W}_{\max }$, then in any layer $\mathcal{G}_{w}$ where $w>\mathcal{W}_{\ell}$ link $\ell$ simply doesn't appear.

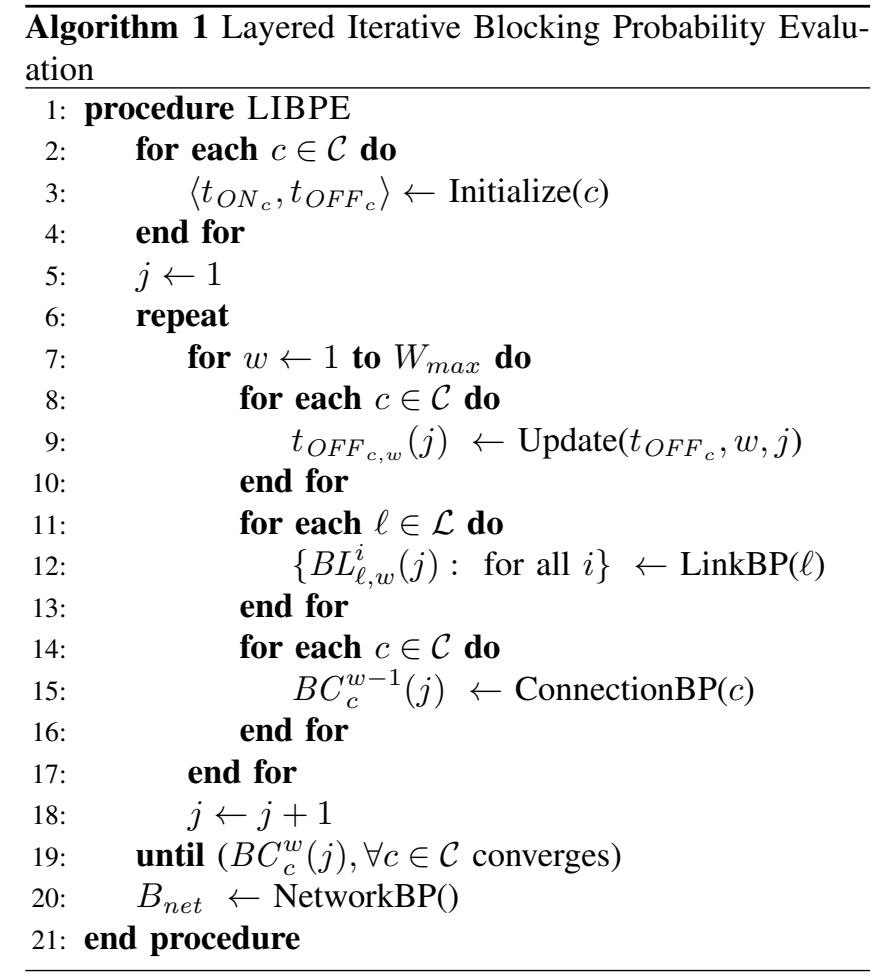

\section{NUMERICAL ILUSTRATIONS}

In this section we illustrate the accuracy of the approximation scheme of LIBPE, by comparing its output with the overflow traffic based method proposed on [13] (denominated as "Overflow" method) and to the result of simulation.

The simulation results where obtained by an event based montecarlo simulation. The simulation was made to represents how dynamic optical networks performs, i.e. it does not use a layer based approach to function, but emulates the network operation without wavelength conversion. Since we wanted to have a good idea about the accuracy of the approximation, we stopped the simulation when we had a statistical relative error on the targets (the global network blocking probabilities) less than 5\%, a pretty stressing objective. We are considering here a typical network performance evaluation process where the systems are considered in equilibrium, so, the simulation results were obtained by removing the initial transient phase, that is, using the concept of warm-up time. For each simulation, enough bursts were generated in order to accomplish a $95 \%$ interval confidence.

In real optical infrastructures, the lengths of ON periods are proportional to the burst size, which depends on the burst aggregation mechanism [27], [28]. These bursts have a maximum size and in this context where resources are scarce, the maximum burst size will in general be reached. This suggests that a constant length ON period is much more realistic than an Exponentially distributed one. Thus, we used the same data but changed the length in time of ON periods of every connection, from random and Exponentially distributed to deterministic, hence constant, ones. 

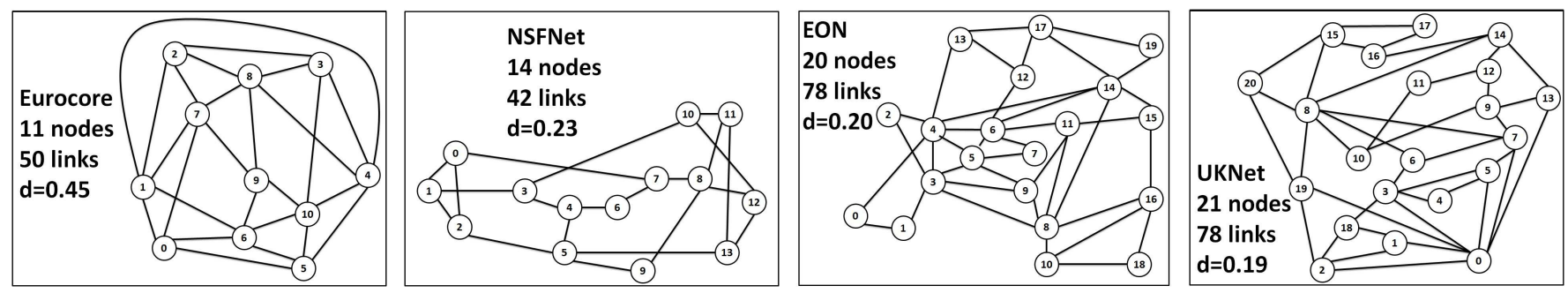

Figure 3. Mesh networks evaluated. Each edge on the networks are bidirectional, so the number of links $L$ refers to unidirectional arcs on each graph. The parameter $d$ is a measure of density: if the graph has $L$ arcs and $N$ nodes, then $d=L /(N(N-1))$.

Eurocore blocking Probability
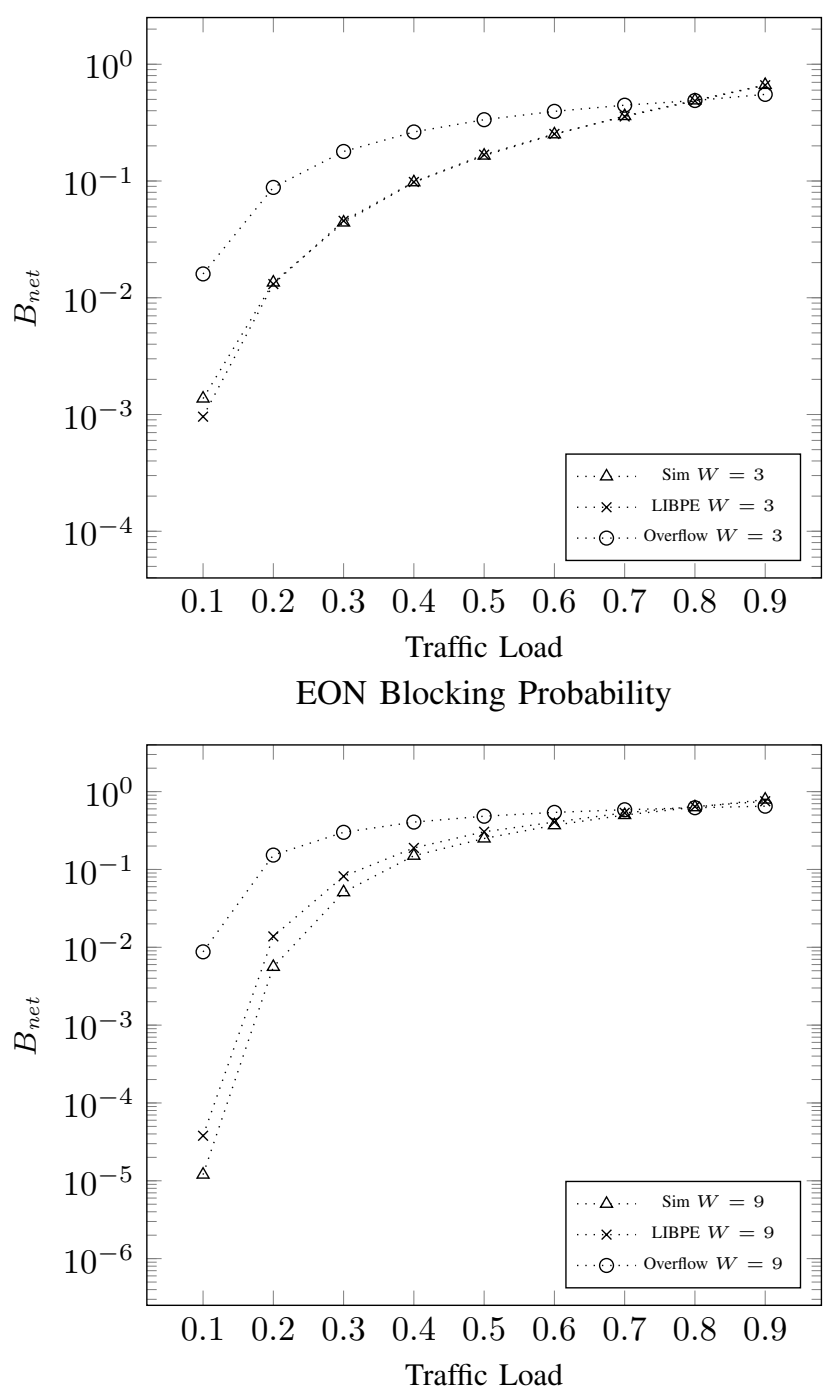

NSFNet Blocking Probability

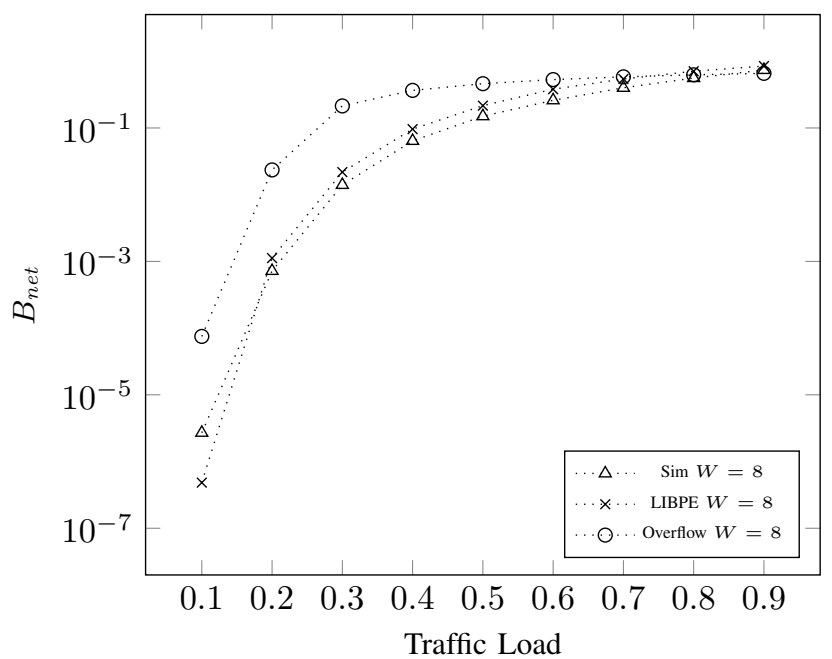

UKNet Blocking Probability

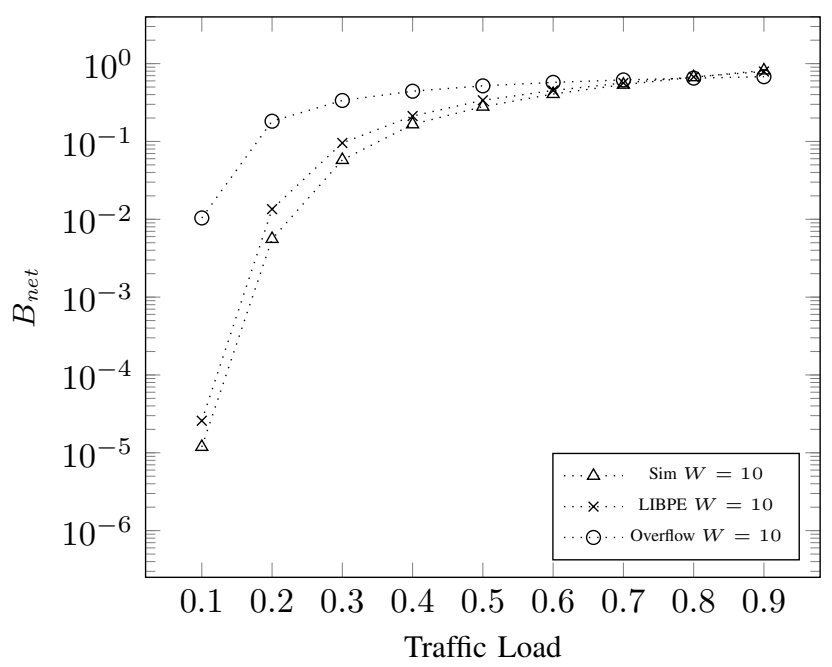

Figure 4. Network blocking probability $B_{\text {net }}$, for Eurocore, NSFNet, EON and UKNet real mesh network topologies and different numbers of wavelengths for each topology, for different connection traffic loads.

Regarding the Overflow method [13], as explained on Section [, it is based on the overflow traffic analysis used on ITU's teletraffic engineering [20]. In this case, the network is split into several layers (one for each wavelength) where the blocked traffic in one layer is overflowed to the next, and uses the Fredericks and Hayward's approximation [20], [21] to transform the bursty overflowed traffic (non-Poisson) into a Poisson flow. Then, to solve each layer blocking probability applies the Erlang-B formula.

On the experiments we compared the outputs of LIBPE and Overflow method with simulation results using four 
different real mesh topologies. Figure 3 shows four of these topologies and some of their parameters: number $N$ of nodes, number $L$ of links (the arcs of the graph) and network density $d=L /(N(N-1))$.

Figure 4 shows the results on the different graphs. The parameter $t_{O N}$ was set to $10 \mathrm{~ms}$ and $t_{O F F}$ was varied, in order to obtain different values for the traffic load, $\varrho$, in the range $[0.1 \ldots 0.9]$.

\section{A. Analysis of the results}

In the experiments, our method always gave results very close to those coming from simulations, as illustrated in the examples used in the paper. We see that our heuristic procedure LIBPE is extremely accurate, and the results reported show that LIBPE is very robust with respect to the model assumptions concerning the length of $\mathrm{ON}$ periods at the sources, the mechanism in which we capture the overflow between network layers, the blocking probability evaluation method at each layer, and using Kelly's to consider the Streamline effect. On the other hand, the Overflow method does not perform as accurately as our method. Therefore, we consider our method performs accurately since we consider in our model correct assumptions and hypothesis.

If we compare the LIBPE and Simulation sets of curves composing Figure 4, it's hard to see any difference between them (in a logarithmic scale, as shown on Fig. 4). Then, to make a zoom on this aspect of the work, consider the case of the UKNet topology with 10 wavelengths per network link, when the load of all connections is 0.3. In this case we observed the largest differences between LIBPE and simulation. Our method obtains a global network blocking probability of $9.56 \cdot 10^{-2}$. and using simulation, the estimation was equal to $5.78 \cdot 10^{-2}$. At an opposite situation, consider the Eurocore network topology with 3 wavelengths per link and, again, a traffic load of 0.3. LIBPE obtains $B_{n e t}=4.56 \cdot 10^{-2}$; and simulation led to a global blocking probability of $4.41 \cdot 10^{-2}$. As a supplementary and positive comment, we always observed that LIBPE evaluates the global blocking probability pessimistically, that is, it provides values slightly higher than those coming from simulating the network. This is a good feature (it allows to guarantee the QoS when solving hig order problems), even if for the moment we don't see what is the exact reason explaining this fact.

The main difference between LIBPE and simulation is the time required to obtain the network blocking probability. For example, to generate the curves for the UKNet network, the mathematical method took less than a second, while the simulation procedures needed about 6 hours in the same computer. The practical implication of this is that our method can be used as an engine internal to a real time decision system, or to assess network performance under different scenarios as a part of an optimization process.

\section{Summary AND EXAMPLE OF APPLICATION}

\section{A. Summary of the paper's proposal}

In qualitative terms, the main characteristics of our approach are the following ones:

- Our method divides the network into $W$ networks $\mathcal{G}_{1}, \ldots, \mathcal{G}_{W}$, that we call layers, to handle the wavelength continuity problem. This allows designing an evaluation technique that decomposes the problem into two different procedures: one for analyzing each layer in isolation, and the other one to interconnect what happens in the different networks. This approach leads to a significant simplification of the evaluation, compared to the effort in computing exactly the blocking probabilities, out of reach even for small networks because of the combinatorial explosion problem.

- To consider the overflowed traffic from a layer to the next, we modify the mean OFF time period for each connection (user) "seen" on each layer, considering the existent dependencies between them.

- The Markov chain proposed to evaluate every link blocking probability on each $\mathcal{G}_{w}$ network considers an ON-OFF traffic, taking account the mean OFF and ON time periods "seen" on each network.

- This method takes into account the fact that the blocking probability of a link depends on what happens with other links in the network ("Streamline Effect"). The majority of the connections pass through more than one link, which means that if a connection request is blocked in one of the first links of its route, the request does not reach the following links of the route ("downstream" links). This phenomenon affects the load perceived by downstream links and therefore their blocking probabilities.

- The model allows heterogeneous traffic. This is important because it represents reality more accurately than the the usual homogeneous traffic assumption.

- Our methodology allows to obtain very accurate results in, say, less than a second for real mesh topologies.

\section{B. Wavelength Dimensioning}

We illustrate the use of the procedure proposed in this paper to dimension the number of wavelengths required on every network link, as a function of the offered traffic and a performance objective The latter typically consists in finding the capacity of the links such that the blocking probability $B C_{c}$ of each connection $c$ does not exceed a predefined maximum tolerance value (typically agreed in the Service Level Agreement between carriers and customers). The number of wavelengths impacts significantly the network cost, therefore an efficient dimensioning of every link in an optical network is of paramount importance. Usually, the dimensioning of these networks has been done based on simulation. The calculation speed is important, so the network designers may solve higher order problems faster.

For different reasons, the usual dimensioning procedures consider homogeneity in the links' capacities, that is, look 


\begin{tabular}{lllll} 
Network Topology & $B C_{c}^{\text {TARGET }}$ & Method & $C_{n e t}$ & Execution Time(s) \\
\hline \multirow{3}{*}{ Eurocore } & $10^{-6}$ & AnHD & 400 & $3.20 \cdot 10^{-2}$ \\
& $10^{-6}$ & SimHD & 400 & $3.98 \cdot 10^{2}$ \\
& $10^{-3}$ & AnHD & 300 & $1.60 \cdot 10^{-2}$ \\
& $10^{-3}$ & SimHD & 300 & $2.28 \cdot 10^{2}$ \\
\hline \multirow{2}{*}{ NSFNet } & $10^{-6}$ & AnHD & 672 & $2.34 \cdot 10^{-1}$ \\
& $10^{-6}$ & SimHD & 672 & $1.21 \cdot 10^{3}$ \\
& $10^{-3}$ & AnHD & 546 & $1.72 \cdot 10^{-1}$ \\
& $10^{-3}$ & SimHD & 546 & $7.25 \cdot 10^{2}$ \\
\hline \multirow{2}{*}{ EON } & $10^{-6}$ & AnHD & 1716 & $1.25 \cdot 10^{0}$ \\
& $10^{-6}$ & SimHD & 1716 & $2.06 \cdot 10^{3}$ \\
& $10^{-3}$ & AnHD & 1404 & $8.90 \cdot 10^{-1}$ \\
& $10^{-3}$ & SimHD & 1404 & $1.82 \cdot 10^{3}$ \\
\hline $10^{-6}$ & AnHD & 1872 & $1.62 \cdot 10^{0}$ \\
& $10^{-6}$ & SimHD & 1872 & $2.37 \cdot 10^{3}$ \\
$10^{-3}$ & AnHD & 1560 & $1.03 \cdot 10^{0}$ \\
& $10^{-3}$ & SimHD & 1560 & $2.11 \cdot 10^{3}$ \\
\hline \multirow{2}{*}{ UKNet } & & & &
\end{tabular}

Table I

COMPUTATIONAL TIME REQUIRED TO CALCULATE THE TOTAL NUMBER OF WAVELENGTHS $C_{n e t}$ WITH THE HOMOGENEOUS DIMENSIONING METHOD BASED ON SIMULATION (SIMHD) AND THE PROPOSED ANALYTICAL PROCEDURE (ANHD). BOTH DIMENSIONING ALGORITHMS CONSIDERS THE MAXIMUM CONNECTION BLOCKING PROBABILITY $B C_{c}^{T A R G E T}$ WITH VALUES EQUAL TO $10^{-3}$ AND $10^{-6}$, AND ARE APPLIED TO EUROCORE, NSFNET, EON AND UKNET REAL MESH NETWORK TOPOLOGIES FOR A MEAN TRAFFIC LOAD EQUAL TO 0.3.

for a capacity $W$, the same on all links, such that the performance objective is reached; see for example [29][33]. For instance, to solve this procedure with different links' capacities, the state space grows exponentially. Then, to solve this problem by simulation becomes hard to obtain. We will then follow here the same approach, because this can facilitate further comparisons with existing methods. The idea is simple: we are given the network topology and the offered traffic, and our tolerance value for the blocking probability of connection $c, B C_{c}^{T A R G E T}$. We then initialize the network capacity $W$ by value 1 and we evaluate the blocking probabilities per connection $B C_{1}, \ldots, B C_{C}$ in two different ways, using simulation and by means of our analytical procedure (in order to be able to compare their respective dimensioning and execution times); then, we check the condition "for all connection $c \in \mathcal{C}, B C_{c} \leq B C_{c}^{T A R G E T \text { ". }}$ If the condition is satisfied, we stop the algorithm. If not, we increase $W$ by 1 and we repeat the procedure.

Let us denote by AnHD the dimensioning procedure using our mathematical approach for evaluating the blocking probabilities, as in Analytical Homogeneous Dimensioning; and by SimHD the analogous method using simulation for the same evaluations, as in Simulation-based Homogeneous Dimensioning. Our goal is to verify the robustness of the approach by checking that both algorithms will produce the similar values for $W$, and the corresponding execution times.

The two dimensioning algorithms, AnHD and SimHD, were applied to different real mesh network topologies. Both algorithms where used in a PC Intel Core I7 with 16GB of RAM and Windows 8 OS. Table $\Pi$ shows the computational time required to calculate the total network $\operatorname{cost} C_{n e t}=$
$L W$, where $W$ is the capacity per link calculated by each algorithm applied to mesh network topologies, for values of $B C_{c}^{T A R G E T}$ equal to $10^{-3}$ and $10^{-6}$, for a mean connection traffic load equal to 0.3 .

In Table [1 it is clear that our method is very accurate. Indeed, in all scenarios evaluated the dimensioning gave the same results of the simulation method. Moreover, it is clear that AnHD has a very low execution time, which is between 3 and 4 orders of magnitude smaller than the execution time of SimHD. For example, on the network EON AnHD is 1648 and 2044 times faster than SimHD, when we consider a maximum blocking probability of $10^{-3}$ and $10^{-6}$ respectively.

\section{CONCLUSiOnS}

In this paper a new layer-based mathematical method called LIBPE for blocking probability evaluation of dynamic WDM optical networks without considering wavelength conversion and taking into account heterogeneous traffic is presented. Another feature of our technique is that it considers that the sources are modeled by $O N-O F F$ processes. This allows to take into account the non-uniform (bursty) nature of the traffic offered to the links. The link blocking dependency is handled by means of the Kelly's Reduced Load method. By dividing the network into several layers, the wavelength continuity constraint can be efficiently taken into account. The interactions between layers is considered through the mean $O F F$ periods seen on every network layer.

The results obtained have been compared against simulation and another commonly used method. The results of 
the proposed technique are accurate enough to closely match the curves obtained by simulation. By its analytical nature, our method allows to obtain the blocking probability of the network in a fraction of a second. This is usually several orders of magnitude faster than using simulation.

As an illustration of the calculating procedure, we report its use for wavelength dimensioning providing a QoS guarantee on the connections' blocking probability. By using our method, we can solve this problem 4 orders of magnitude faster than simulation on the scenarios presented. This is relevant because allows the network designer to solve problems of higher order several times, to adjust and improve the network designing procedures.

As in several procedures of this type, some mathematical aspects have not been considered, namely the existence and unicity of fixed points, or the validity of the convergence tests in the fixed point calculations. In future work we will explore these issues, for instance, by analyzing the potential use of Brouwer theorem and extensions for the analysis of the fixed points, or the capabilities of different convergence tests. Our only claim here is the fact that in a large set of experiments, some of which are reported here, we never found any problem with these issues.

\section{ACKNOWLEDGMENTS}

This work received financial support from FONDEF ID14I10129, Stic/Amsud-Conicyt project “DAT", USM PIIC Proyect, CONICYT $\mathrm{PhD}$ and CONICYT PhD internship scholarships. These projects and institutions are then gratefully acknowledged.

\section{REFERENCES}

[1] A. A. M. Saleh and J. M. Simmons, "Technology and architecture to enable the explosive growth of the internet," Communications Magazine, IEEE 49, 126-132 (2011).

[2] A. Sano, T. Kobayashi, S. Yamanaka, A. Matsuura, H. Kawakami, Y. Miyamoto, K. Ishihara, and H. Masuda, "102.3-tb/s (224 x 548$\mathrm{gb} / \mathrm{s}) \mathrm{c}$ - and extended l-band all-raman transmission over $240 \mathrm{~km}$ using pdm-64qam single carrier fdm with digital pilot tone," in "Optical Fiber Communication Conference and Exposition (OFC/NFOEC), 2012 and the National Fiber Optic Engineers Conference," (2012), pp. 1-3.

[3] R. Ramaswami, K. Sivarajan, and G. Sasaki, Optical Networks: A Practical Perspective, 3rd Edition (Morgan Kaufmann Publishers Inc., San Francisco, CA, USA, 2009), 3rd ed.

[4] A. Mokhtar and M. Azizoğlu, "Adaptive wavelength routing in alloptical networks," IEEE/ACM Trans. Netw. 6, 197-206 (1998).

[5] B. Mukherjee, Optical WDM networks (Springer Science \& Business Media, 2006)

[6] A. Zapata-Beghelli and P. Bayvel, "Dynamic versus static wavelengthrouted optical networks," Lightwave Technology, Journal of 26, 3403 3415 (2008).

[7] M. Düser, A. Zapata, and P. Bayvel, "Investigation of the scalability of dynamic wavelength-routed optical networks," J. Opt. Netw. 3, 674693 (2004).

[8] A. Alyatama, "Computing blocking probabilities in survivable wdm optical networks," Photonic Network Communications 27, 34-46 (2014).

[9] A. Alyatama, "Wavelength decomposition approach for computing blocking probabilities in multicast wdm optical networks," Opt. Switch. Netw. 12, 24-33 (2014).

[10] J. Triay, C. Cervelló-Pastor, and V. M. Vokkarane, "Analytical blocking probability model for hybrid immediate and advance reservations in optical wdm networks," IEEE/ACM Trans. Netw. 21, 1890-1903 (2013).
[11] V. Abramov, S. Li, M. Wang, E. Wong, and M. Zukerman, "Computation of blocking probability for large circuit switched networks," Communications Letters, IEEE 16, 1892-1895 (2012).

[12] J. He, M. Brandt-Pearce, and S. Subramaniam, "Analysis of blocking probability for first-fit wavelength assignment in transmissionimpaired optical networks," J. Opt. Commun. Netw. 3, 411-425 (2011).

[13] A. Alyatama, "Wavelength decomposition approach for computing blocking probabilities in WDM optical networks without wavelength conversions," Computer Networks 49, 727 - 742 (2005).

[14] K. Lu, G. Xiao, and I. Chlamtac, "Analysis of blocking probability for distributed lightpath establishment in wdm optical networks," Networking, IEEE/ACM Transactions on 13, 187-197 (2005).

[15] A. Sridharan and K. Sivarajan, "Blocking in all-optical networks," Networking, IEEE/ACM Transactions on 12, 384-397 (2004).

[16] R. Vallejos, J. Olavarría, and N. Jara, "Blocking evaluation and analysis of dynamic WDM networks under heterogeneous on/off traffic," Optical Switching and Networking 20, 35 - 45 (2016).

[17] L. H. Bonani and I. E. Fonseca, "Estimating the blocking probability in wavelength-routed optical networks," Optical Switching and Networking 10, 430 - 438 (2013).

[18] Y. Zhao and J. Zhang, "Blocking probability analysis model for flexible spectrum optical networks," Chin. Opt. Lett. 12, 070601 (2014).

[19] R. Almeida, D. Campelo, H. Waldman, and K. Guild, "Accounting for link load correlation in the estimation of blocking probabilities in arbitrary network topologies," Communications Letters, IEEE 11, 625-627 (2007).

[20] B. Iversen Villy, "Teletraffic engineering handbook," (2002).

[21] A. A. Fredericks, "Congestion in blocking systems-a simple approximation technique," Bell System Technical Journal 59, 805-827 (1980).

[22] T. Bonald, "The erlang model with non-poisson call arrivals," SIGMETRICS Perform. Eval. Rev. 34, 276-286 (2006).

[23] J. Zhang, Y. Peng, E. W. Wong, and M. Zukerman, "Sensitivity of blocking probability in the generalized engset model for obs," Communications Letters, IEEE 15, 1243-1245 (2011).

[24] S. Ross, Stochastic Processes (John Wiley \& Sons, Inc., 1996), 2nd ed.

[25] Q. Chen, G. Mohan, and K. C. Chua, "Route optimization in optical burst switched networks considering the streamline effect," Computer Networks 52, 2033 - 2044 (2008). Challenges and Opportunities in Advanced Optical Networking.

[26] F. P. Kelly, "Loss networks," The Annals of Applied Probability 1 , pp. 319-378 (1991).

[27] A. Ge, F. Callegati, and L. S. Tamil, "On optical burst switching and self-similar traffic," Communications Letters, IEEE 4, 98-100 (2000).

[28] Y. Xiong, M. Vandenhoute, and H. C. Cankaya, "Control architecture in optical burst-switched wdm networks," Selected Areas in Communications, IEEE Journal on 18, 1838-1851 (2000).

[29] E. Kozlovski, M. Duser, I. De Miguel, and P. Bayvel, "Analysis of burst scheduling for dynamic wavelength assignment in optical burstswitched networks," in "IEEE, Proc. LEOS,", vol. 1 (2001), vol. 1.

[30] T. K. Nayak and K. N. Sivarajan, "A new approach to dimensioning optical networks," IEEE Journal on Selected Areas in Communications 20, 134-148 (2002).

[31] J. Teng and G. N. Rouskas, "Routing path optimization in optical burst switched networks," in "Proc. ONDM,", vol. 2005 (Citeseer, 2005), vol. 2005.

[32] J. Yu, I. Yamashita, S. Seikai, and K. Kitayama, "Upgrade design of survivable wavelength-routed networks for increase of traffic loads," in "Conference onOptical Network Design and Modeling, 2005.", (2005), pp. 163-174.

[33] A. L. Chiu, G. Choudhury, G. Clapp, R. Doverspike, J. W. Gannett, J. G. Klincewicz, G. Li, R. A. Skoog, J. Strand, A. V. Lehmen, and D. Xu, "Network design and architectures for highly dynamic next-generation ip-over-optical long distance networks," Journal of Lightwave Technology 27, 1878-1890 (2009). 